\title{
Lesões dermatológicas em pacientes infectados pelo vírus linfotrópico humano de células T do tipo 1 (HTLV-1)
}

\author{
Dermatologic lesions in patients infected with the human \\ T-cell lymphotropic vírus type 1 (HTLV-1)
}

\author{
Vandack Nobre', Antônio Carlos Martins Guedes², Fernando Augusto Proietti ${ }^{3}$, Edel Stanciolli ${ }^{4}$, \\ Marina Lobato Martins ${ }^{5}$, José Carlos Serufo ${ }^{1}$, Carlos Maurício Antunes ${ }^{6}$, \\ Maria Aparecida Grossi ${ }^{7}$, José Roberto Lambertucci ${ }^{1}$ \\ e GIPH (Grupo Interdisciplinar de Pesquisas em HTLV-1/2)
}

\begin{abstract}
RESUMO
0 vírus linfotrópico humano de células T do tipo 1 (HTLV-1) é o primeiro retrovírus isolado do ser humano. Descreveuse, em pouco tempo, o seu papel etiológico em algumas doenças, com destaque para a leucemia/linfoma de células T do adulto (ATLL), a mielopatia associada ao HTLV-1/paraparesia espástica tropical (HAM/TSP) e a uveíte associada ao HTLV-1 (HAU) . Na década de 90, o HTLV-1 foi associado a eczema grave da infância, conhecido como dermatite infecciosa (DI). Desde então, diversos outros tipos de lesões cutâneas têm sido observados em pacientes infectados pelo HTLV-1, em especial, nos doentes de HAM/TSP ou de ATL. Porém, mesmo portadores assintomáticos do vírus apresentam doenças dermatológicas. Excetuando-se a dermatite infecciosa, não há lesão da pele específica da infecção pelo HTLV-1. Aqui, os autores apresentam as principais lesões dermatológicas descritas em pacientes infectados pelo HTLV-1, destacando 0 valor epidemiológico e clínico desses achados.
\end{abstract}

Palavras-chaves: HTLV-1. Retrovírus. ATLL HAM/TSP. Lesões dermatológicas. Pele.

\begin{abstract}
Human T-cell Lymphotropic vírus type I (HTLV-1) was the first human retrovírus described. Some time after its discovery a group of diseases were related to this vírus, such as, adult T-cell leukemia lymphoma (ATLL), HTLV-1 associated myelopathy/tropical spastic paraparesis (HAM/TSP) and HTLV-1 associated uveitis (HAU). In the nineties, HTLV-1 was associated to a severe eczema of children, called infective dermatitis (ID). Since then, several other skin manifestations have been observed in HTLV-1-infected individuals, particularly in patients with ATLL or HAM/TSP. However, according to some reports, dermatologic lesions are also common in asymptomatic HTLV-1 carriers. Besides ID, all other skin lesions reported are nonspecific. The aim of this review is to outline the dermatologic manifestations reported in HTLV- 1 infected patients, emphasizing the clinical and epidemiological value of these findings.
\end{abstract}

Key-words: HTLV-1. Retrovírus. ATLL HAM/TSP. Dermatological lesions. Skin.

\footnotetext{
1. Serviço de Doenças Infecciosas e Parasitárias da Faculdade de Medicina da Universidade Federal de Minas Gerais, Belo Horizonte, MG, Brasil. 2. Serviço de Dermatologia da Faculdade de Medicina da Universidade Federal de Minas, Belo Horizonte, MG, Brasil. 3. Departamento de Medicina Social e Preventiva da Faculdade de Medicina da Universidade Federal de Minas Gerais, Belo Horizonte, MG, Brasil. 4. Departamento de Microbiologia do Instituto de Ciências Biológicas da Universidade Federal de Minas Gerais, Belo Horizonte, MG, Brasil. 5. Fundação Hemominas, Belo Horizonte, MG, Brasil. 6. Departamento de Parasitologia do Instituto de Ciências Biológicas da Universidade Federal de Minas Gerais, Belo Horizonte, MG, Brasil. 7. Centro Geral de Pediatria (CGP), FHEMIG, Belo Horizonte, MG, Brasil.

Endereço para correspondência: Prof. José Roberto Lambertucci. Faculdade de Medicina/UFMG. Avenida Alfredo Balena 190, 30130-100 Belo Horizonte, MG, Brasil.

e-mail: lamber@uai.com.br

Recebido para publicação em 01/10/2004

Aceito em 2/10/2004
} 
0 vírus linfotrópico humano de células T do tipo 1 (HTLV-1) foi o primeiro retrovírus isolado no ser humano. As primeiras descrições desse vírus ocorreram na década de 80, nos Estados Unidos ${ }^{79}{ }^{80} \mathrm{e}$ no Japãa ${ }^{38}{ }^{39} \mathrm{e}$, desde então, novos conhecimentos acerca da infecção por esse agente surgiram ${ }^{16}{ }^{65}$. Adescrição inicial do vírus linfotrópico humano de células T do tipo 2 (HTLV-2) aconteceu em 1982, em paciente com leucemia de células pilosas ${ }^{42}$. Informações adicionais sobre o HTLV-2 podem ser obtidas em outro estud ${ }^{34}$.

0 HTLV-1 pertence à família retroviridae, subfamília oncovirina $e^{19}$. Asua transmissão ocorre predominantemente por via vertical, com destaque para a amamentação ${ }^{41}$. Outras formas de transmissão são a transfusão de sangue e de hemoderivados contaminados com 0 vírus, a relação sexual ( sobretudo do homem para a mulher) e 0 uso de drogas injetáveis ${ }^{1665}$. 0 HTLV-1 é um vírus cosmopolita, apresentando ampla distribuição mundial ${ }^{62}$. Entre as regiões de maior endemicidade destacam-se 0 Japão, a Melanésia, o Caribe, a América do Sul e a África 161739408489 . A maior parte dos estudos de prevalência realizados considerou doadores de sangue ou outros grupos com risco elevado para adquirir a infecção, como usuários de drogas injetáveis e mulheres profissionais do sexo. São escassos, portanto, os estudos de base populacional ${ }^{25}{ }^{83}$. 0 Brasil apresenta prevalência moderadamente elevada para o HTLV-1, observando-se aumento da prevalência, quando se caminha a partir dos estados do sul para os da região norte do país (Catalan-Soares, comunicação pessoal). Considerando-se a sua população, acredita-se que o Brasil abrigue o maior número absoluto de indivíduos infectados pelo HTLV-1 no mund $0^{83}$.

0 diagnóstico da infecção pelo HTLV-1 é feito geralmente por meio de exames sorológicos, com destaque para as técnicas de ELISA ( triagem) e Western blot ( confirmatório) ${ }^{20}$. 0 Western blot permite distinguir a infecção pelo HTLV-1 do HTLV-2. Areação em cadeia da polimerase (PCR) no sangue, líquor ou em outros materiais (e.g., pele, linfonodos) pode ser útil, particularmente nos casos em que a sorologia mostra-se indeterminada ${ }^{26}$.

Desde a descrição do HTLV-1, acreditava-se em sua associação com doenças humanas. A primeira condição associada ao vírus foi a leucemia/linfoma de células T do adulto (ATL) , cujo papel etiológico do HTLV-1 foi confirmado com a demonstração da integração monoclonal do DNA proviral ao genoma das células neoplásicas ${ }^{38} 6193103$. Em seguida, definiu-se 0 papel etiológico do HTLV-1 em um tipo de mielopatia espástica, hoje conhecida como mielopatia associada ao HTLV-1 ou paraparesia espástica tropical (HAM/TSP) ${ }^{26} 71$ 84. Finalmente, em 1992, Mochisuchi e colaboradores ${ }^{68}{ }^{69}$ demonstraram associação causal do HTLV-1 a uma forma de uveíte predominantemente intermediária, a uveíte associada ao HTLV-1 (HAU).

Diversos relatos apontam para a presença de lesões dermatológicas em pacientes infectados pelo vírus, em especial, os portadores de ATLZ 259398 e de HAM/TSP3 144959 92. A seguir, constatou-se que as lesões ocorrem também em indivíduos infectados e considerados assintomáticos ${ }^{31}$. 0 estudo dessas dermatopatias merece destaque, uma vez que, a pele permite a realização de procedimentos diagnósticos de doenças que acometem os órgãos internos ${ }^{48}$.
No início da década de 90, La Grenade e cols ${ }^{49} 51545657$ correlacionaram 0 HTLV-1 a um eczema grave da infância, descrito na Jamaica em $1966^{105}$ e conhecido como dermatite infecciosa (DI). Ainda hoje, essa constitui a única dermatopatia específica da infecção pelo HTLV-1. A seguir, serão descritas as principais lesões dermatológicas observadas em pacientes infectados pelo HTLV-1.

\section{CLASSIFICAÇÃo}

Nã̃o há classificação consensual das lesões dermatológicas apresentadas pelos pacientes infectados pelo HTIV-1, ainda que as propostas se mostrem semelhantes. Nesta revisão, utilizou-se uma classificação que aglutina as sugeridas por Rueda \& Blanck ${ }^{87}$ e por La Grenade ${ }^{53}$, e que expressa mais adequadamente 0 conhecimento atual sobre a doença e 0 entendimento dos autores deste trabalho acerca dos prováveis mecanismos subjacentes a essas lesões (Tabela 1).

Tabela 1 - Classificações das lesões dermatológicas, segundo três autores, em pacientes infectados pelo HILV-1.

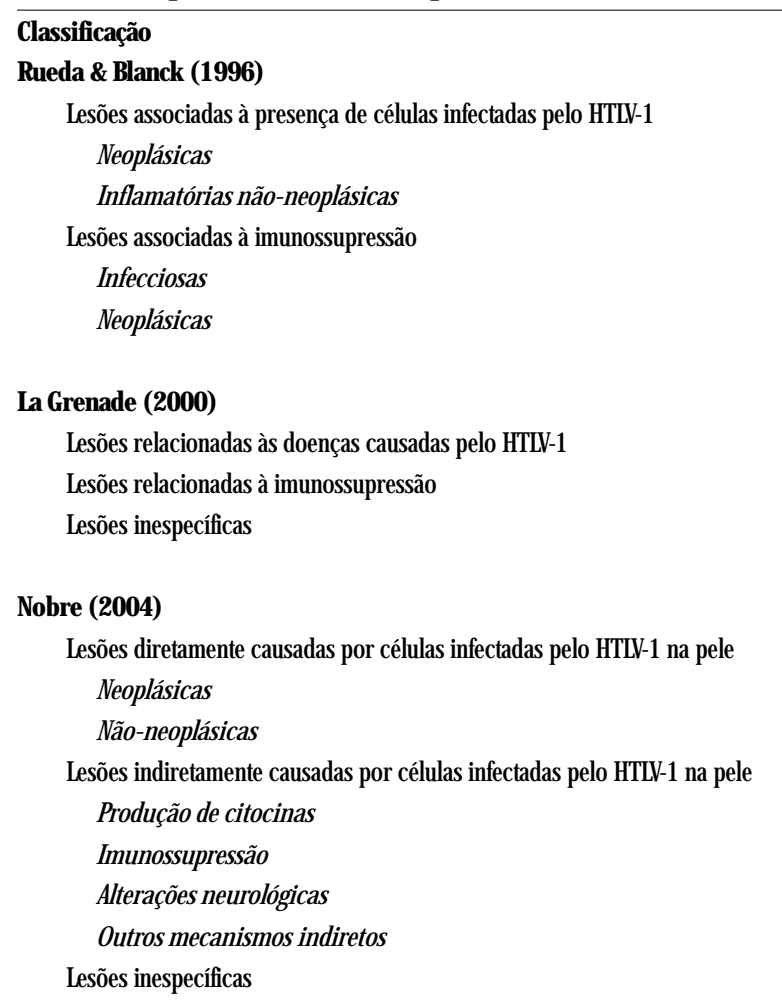

1- Lesões diretamente causadas por células infectadas pelo HTLV-1: acredita-se que a maior parte das lesões cutâneas vistas nos portadores do HTLV-1 seja associada à presença de células infectadas na pele. As lesões podem ser determinadas de forma direta ou indireta. No primeiro caso ocorre, por exemplo, infiltração cutânea de células neoplásicas, como se observa nos pacientes com ATLL 0 estudo histológico da pele desses pacientes revela densos infiltrados de células pleomórficas (linfócitos atípicos) na derme que, com frequêencia, se estendem ao tecido subcutâneo. 0 achado de epidermotropismo é incomum, em contraposição ao que se observa nos linfomas 
cutâneos de células T ( LCCT) não associados ao HTLV-1 ${ }^{45}$. Além disso, o HTLV-1 pode infectar e, hipoteticamente, modificar a função de células constituintes do tecido cutâneo, tais como, ceratinócitos, fibroblastos e células de glândulas sudoríparas ${ }^{467890}$.

2 - Lesões indiretamente causadas por células infectadas pelo HTLV-1: há evidências de que citocinas produzidas por linfócitos infectados pelo HTLV-1 determinem alterações funcionais de células constituintes da pele, como, por exemplo, dos ceratinócitos ${ }^{67}$. Esse mecanismo, ainda pouco estudado em relação às lesões cutâneas, parece bastante plausivel, já que predomina em outras condições associadas ao HTLV-1, mormente na HAM/TSP ${ }^{85}$.

Outro grupo de lesões indiretas associa-se à imunossupressão. Os pacientes portadores de ATL apresentam imunossupressão grave e, por conseguinte, várias lesões oportunistas podem se desenvolver, citando-se a pneumocistose, a criptococose e 0 sarcoma de Kaposi ${ }^{66}$. Entretanto, mesmo pacientes sem ATL, apresentam imunossupressão, sobretudo do tipo celular. Nesse sentido, Tachibana e colaboradores ${ }^{97}$ demonstraram que, os pacientes infectados pelo HTLV-1 apresentam diminuição da resposta cutânea tardia ao PPD. Popovic e colaboradores ${ }^{81}$ evidenciaram significativas alterações na função de linfócitos T auxiliares e de linfócitos citotóxicos promovidas pelo HTLV-1. № primeiro caso, quando infectadas, as células T podem estimular os linfócitos B de forma anômala, promovendo produção policlonal de imunoglobulinas, independente da estimulação antigênica. Com relação aos linfócitos citotóxicos, observou-se que à medida que a proporção de células infectadas pelo HTLV-1 aumenta, a atividade citotóxica dessas células diminui. Finalmente, estudando 0 índice de regressão do foco de células B induzidas pelo vírus Epstein Barr, Katsuki e colaboradores ${ }^{44}$ demonstraram que, os portadores assintomáticos do HTLV-1 apresentam supressão da imunidade celular. Outras evidências da presença de imunossupressão nos pacientes infectados pelo HTLV-1, são: a) aumento da prevalência e da gravidade da estrongiloidíase e da escabiose nesses pacientes ${ }^{51} 587177$ 82; b) desenvolvimento da dermatite infecciosa (DI) , cuja associação com o HTLV-1 encontrase estabelecida ${ }^{54}{ }^{56} \mathrm{e}, \mathrm{c}$ ) a apresentação at́pica de doenças cutâneas em pacientes infectados pelo HTLV-1, como ocorre na sífilis secundária ${ }^{15}$.

Outros mecanismos existem. Assim, as alterações, do tipo xerose ( secura na pele), observadas nos pacientes com HAM/ TSP, devem-se a alterações do sistema nervoso autônomo que alteram a função de glândulas sudoríparas responsáveis pela lubrificação da pele ${ }^{36}$.

3- Lesões inespecíficas: grande parte das lesões dermatológicas observadas nos pacientes infectados pelo HTLV1 não conhecem explicação. Essas lesões são, portanto, consideradas inespecíficas. Cita-se, como exemplo, 0 vitiligo.
As principais lesões dermatológicas apresentadas pelos pacientes infectados pelo HTLV-1 encontram-se listadas na Tabela 2.

Tabela 2 - Principais lesões dermatológicas descritas nos pacientes infectados pelo HILV-1.

\begin{tabular}{|c|c|c|}
\hline $\begin{array}{l}\text { Característica } \\
\text { da infecção }\end{array}$ & Lesões dermatológicas & Observações \\
\hline 1- ATL & $\begin{array}{l}\text { Máculas, pápulas, placas, } \\
\text { nódulos, eritrodermias, } \\
\text { tumorações, dermatofitose, } \\
\text { escabiose. }\end{array}$ & $\begin{array}{l}\text { Predominam na forma subaguda } \\
\text { da doença. Podem ser difusas ou } \\
\text { localizadas. Existea forma cutânea } \\
\text { isolada da ATL. Dermatopatias } \\
\text { podem ser pródromos da ATL }\end{array}$ \\
\hline 2- HAM/TSP & $\begin{array}{l}\text { Xerose, ictiose, candidíase, } \\
\text { eritemapalmar emalar, lesões } \\
\text { relacionadas à imobilidade } \\
\text { (escaras, dermatite perineal). } \\
\text { Cita-se também dermatofitose } \\
\text { e foliculite decalvante. }\end{array}$ & $\begin{array}{l}\text { As lesões predominantes são: } \\
\text { xerose e ictiose. }\end{array}$ \\
\hline $\begin{array}{l}\text { 3- Dermatite } \\
\text { infecciosa (DI) }\end{array}$ & $\begin{array}{l}\text { Eczema grave e recidivante, } \\
\text { predomina em crianças, } \\
\text { acomete várias regiões do } \\
\text { corpo. }\end{array}$ & $\begin{array}{l}\text { Pode ocorrer em adultos jovens } \\
\text { e geralmente está associada à } \\
\text { infecção persistente por cocos } \\
\text { Gram positivos. } 0 \text { principal } \\
\text { diagnóstico diferencial é a } \\
\text { dermatite seborréica. DI pode } \\
\text { ser pródromo de ATL ou de } \\
\text { HAM/TSP }\end{array}$ \\
\hline $\begin{array}{l}\text { 4- Portadores } \\
\text { assintomáticos* }\end{array}$ & $\begin{array}{l}\text { Xerose, ictiose, dermatite } \\
\text { seborréica, vitiligo, escabiose, } \\
\text { dermatofitoses }\end{array}$ & $\begin{array}{l}\text { Poderiam motivar a suspeição da } \\
\text { infecção pelo HTLV-I. }\end{array}$ \\
\hline
\end{tabular}

*Sem ATLL HAM/TSP

1- Pacientes com ATLL: diferentemente dos LCCT não associados ao HTLV-1, geralmente primários da pele, na ATLL 0 processo neoplásico mais comumente se inicia nos órgãos internos, atingindo a pele apenas numa segunda fase. Cerca de $40 \%$ a 50\% dos pacientes com ATLL apresentam lesões dermatológicas ${ }^{93} 1011^{102}$. Estas se mostram variadas e inespecíficas, predominando nos pacientes com a forma subaguda da doença. Assim, podem ocorrer máculas, pápulas, nódulos, placas, eritrodermia e tumorações, sendo as três primeiras mais comuns ${ }^{8}{ }^{93}$. As lesões podem ser difusas ou localizadas. Dosaka e colaboradores ${ }^{25}$ descreveram as dermatoses encontradas em nove pacientes com ATLL em diferentes fases de evolução. Dentre as lesões descritas cita-se a verruga vulgar, os tumores do subcutâneo, 0 eritema nodoso, as pápulas, nódulos, eritema reticular, prurido, Tinea corporis e eritrodermia esfoliativa. As lesões também podem variar ao longo do curso da ATL ${ }^{98}$.

Há, adicionalmente, a forma cutânea isolada da ATLL, caracterizada pela integração monoclonal do DNA proviral ao genoma dos linfócitos que infiltram a pele, e policlonal nos linfócitos do sangue periférico ${ }^{28,29}$. A distinção dessa forma com os LCCT não associados à ATLL (micose fungóide e síndrome de Sézary) é difícili ${ }^{45}$. Quanto à micose fungóide, há grande discussão na literatura acerca do papel etiológico do HTLV-1 na gênese desta neoplasia. Ainda que a pesquisa sorológica do HTLV-1 revele-se positiva em apenas $15 \%$ dos $\operatorname{casos}^{94}$, alguns grupos relatam 0 encontro de material genético do vírus na pele de praticamente todos os pacientes com micose fungóide $e^{46} 747576$. Contudo, os 
achados de outros pesquisadores, sobretudo europeus, negam tal associaçãa ${ }^{41047} 62$ 106. Bittencourte colaboradores ${ }^{8}$ relataram 0 primeiro caso de linfoma cutâneo associado ao HTLV-1 ocorrido na Bahia. 0 paciente apresentava massas tumorais na região cervical e lesões maculopapulares descamativas no tronco e membros. Em outro estudo, 0 mesmo grupo não observou diferenças significativas na avaliação histológica da pele de pacientes portadores de LCCT associados ou não ao HTLV-17.

A ATLL é dividida em cinco subtipos, quais sejam, as formas aguda, subaguda ou smouldering, crônica, o tipo linfoma e a doença cutânea isolada ${ }^{93}$. As principais lesões dermatológicas observadas em cada subtipo são ${ }^{87}$ :

a) ATLLaguda: predominam lesões nodulares e papulares.

b) ATLL crônica: predominam placas eritematosas e edematosas. Eritrodermia ocorre com menor freqüência.

c) ATL subaguda ou smouldering: é a forma que mais se associa a lesões cutâneas. Estas se assemelham às observadas nas outras formas de ATL, predominando nódulos e pápulas.

d) ATL tipo linfoma: as lesões são extensas, evoluindo de placas para nódulos e tumores.

e) ATLL cutânea isolada: representa 0 linfoma cutâneo associado ao HTLV-1. As lesões mais comuns são tumores, pápulas, nódulos e eritrodermia.

Há várias publicações na literatura sugerindo que lesões cutâneas podem se comportar como pródromos das doenças associadas ao HTLV-1, notadamente da ATLL 13223791105. Entretanto, a maioria dos trabalhos trata de casos isolados. A dermatite infecciosa constitui a dermatopatia mais bem estudada nesse sentido, ainda que, mesmo neste caso, paire dúvidas acerca do caráter pré-maligno das lesões ${ }^{30} 351$.

2- Pacientes com HAMTSP: várias lesões cutâneas têm sido observadas em pacientes portadores de HAM/TSP. Hashiguchi e colaboradores ${ }^{36}$ observaram a presença de xerodermia em 12 (66\%) de 18 pacientes com HAM/TSP no Japão, a maior parte deles com sinais de hipo-hidrose. Numa outra série de casos, os mesmos autores relataram 0 encontro de eritema palmar e malar em 19 (22,9\%) de 83 pacientes com HAM/TSP.

Mais recentemente, no Brasil, Lenzi e colaboradores ${ }^{60}$ estudaram as manifestações cutâneas apresentadas por 60 pacientes com HAM/TSP, comparando-os com 38 doadores de sangue sem infecção pelo HTLV-1, pareados por idade. Xerodermia, candidíase cutânea e eritema palmar mostraramse significativamente mais comuns nos pacientes com HAM/ TSP. Os autores observaram que a intensidade da xerose se correlacionava diretamente com a gravidade da doença neurológica.

Outras lesões descritas nos pacientes com HAM/TSP são eritrodermia ${ }^{14}{ }^{49}$, foliculite decalvante ${ }^{4}$, dermatofitose, dermatite seborréica, vitiligo, molusco contagioso e erisipela bolhosa de repetição ${ }^{14}$.
3- Dermatite infecciosa (DI) : a DI foi descrita por Sweet em $1966^{96}$. No ano seguinte, Walshe descreveu casos adicionais da doença e demonstrou a presença de infecção bacteriana persistente na pele e nas mucosas dos pacientes com essa dermatos $\mathrm{e}^{104}$. A DI representa 0 terceiro tipo de eczema mais freqüentemente encontrado em crianças jamaicanas ${ }^{57}$. Além da Jamaica, há relatos de casos provenientes de outros países endêmicos para 0 HTLV-1, como 0 Japão $0^{100}$ e a Colômbia9. № Brasil, o primeiro caso de DI foi descrito em $1996^{6}$.

A suspeita inicial acerca da associação entre o HTLV-1 e a DI adveio da observação de óbitos por ATLL em mães de pacientes portadores da dermatite e foi confirmada em trabalhos publicados no início da década de 90 . No primeiro estudo, os autores demonstraram soropositividade para 0 vírus em 14 crianças com DI. Onze crianças portadoras de eczema atópico foram avaliadas como controles e todas apresentaram sorologia negativa ${ }^{54}$. Alguns anos depois, um grupo maior de pacientes foi estudado, incluindo 50 casos de DI, e todos se revelaram infectados pelo HTLV-1. Por outro lado, nenhum dos 36 controles com eczema atópico apresentaram sorologia positiva ${ }^{50}$.

ADI habitualmente se manifesta após os dois anos de idade. Inicia-se com rinite, em geral interpretada pelos pais como resfriado. Segue-se aumento da secreção nasal e descamação do couro cabeludo, pavilhão auricular, narinas, pescoço, axilas, umbigo e períneo, às vezes associados com blefaroconjuntivite. Febre alta mostra-se incomum. Ocorrem, em seguida, dermatite exsudativa grave, formação de crostas nas narinas e outras regiões, coriza aquosa e intensificação das lesões nas áreas citadas. Linfadenomegalia reacional é comum. A cultura da secreção obtida nas narinas e pele demonstra $\mathrm{S}$. aureus e/ou Streptococcus beta-hemolítico. Os principais diagnósticos diferenciais são a dermatite seborréica e 0 eczema atópico ${ }^{56}$. La Grenade e colaboradores propuseram critérios diagnósticos para a dermatite infecciosa, dividindo-os em maiores e menores ${ }^{55}$. Para se definir um caso são necessários quatro dos cinco critérios maiores, sendo obrigatórios os critérios "1", "2" e "3". Para 0 preenchimento do critério maior "1", pelo menos duas das regiões citadas devem estar acometidas (Tabela 3 ).

Os pacientes portadores de DI apresentam resposta inicial adequada ao tratamento antimicrobiano; contudo, observa-se rápida piora após a interrupção do mesmo. Habitualmente, administram-se os antibióticos por via oral até a puberdade, época em que os sintomas costumam remitir. Anecessidade de se manter a antibioticoterapia por tempo prolongado nos pacientes com dermatite infecciosa favorece a hipótese de imunossupressão como base para o desenvolvimento da doença. Contudo, fatores sócioeconômicos podem contribuir. Corticoesteróides tópicos de leve potência, anti-histamínicos orais e xampus ceratolíticos para 0 couro cabeludo também são indicados. Năo se utiliza terapia antiviral ou imunomoduladora ${ }^{55}$.

Complicações ocorrem em até 35\% dos pacientes com DI, sendo mais comuns, as opacidades corneanas, a escabiose norueguesa, as bronquiectasias e as infecções helmínticas, destacando-se a estrongiloidíase. Além dessas, cita-se a pneumonite intersticial linfocítica, a histiocitose e a glomerulonefrite ${ }^{52}$. 
Tabela 3 - Critérios diagnósticos para dermatite infecciosa (La Grenade et al, 1998).

\section{Critérios}

\section{Critérios maiores}

1. Eczema do couro cabeludo, pavilhão auricular, pálpebras, pele paranasal, pescoço, axilas e/ou região inguinal.

2. Coriza crônica, com formação de crostas nas narinas e ausência de rinite.

3. Sorologia positiva para o HTLV-1

4. Dermatite crônica, rapidamente responsiva aos antibióticos; entretanto, há recidiva precoce após a interrupção do tratamento.

5. Início precoce na infância.

\section{Critérios menores}

1. Rash papular fino generalizado.

2. Cultura de material obtido na região anterior das narinas ou da pele de outros locais positiva para Staphylococcus aureus ou Streptococcus beta-hemolítico

3. Anemia.

4. Elevação da velocidade de eritrossedimentação.

5. Linfadenopatia generalizada com linfadenite dermatopática.

6. Hiperimunoglobulinemia ( $\operatorname{IgD}$ e $\operatorname{IgE}$ ).

7. Elevação das contagens de células CD4+, CD8+ e da razão CD4/CD8.

A seguir, descreve-se um caso de dermatite infecciosa acompanhado por um dos autores (MAG):

A paciente, de dois anos de idade, residente na região metropolitana de Belo Horizonte, Minas Gerais, foi atendida no Centro Geral de Pediatria da Fundação Hospitalar do Estado de Minas Gerais, apresentando lesões eczematosas e pruriginosas, localizadas predominantemente no couro cabeludo e nas regiões perinasal, retro-auricular, axilar e inguino-crural (Figura 1). 0 início das lesões se deu aos três meses de idade e se mostravam freqüentemente associadas a infecção bacteriana secundária. A evolução da paciente caracterizou-se por duas internações prolongadas. Como complicações do quadro, diagnosticou-se desnutrição, otite externa, sinusite e infecção do trato urinário. Durante as internações, a cultura de secreções cutâneas revelou-se positiva para Pseudomonas aeruginosa e Staphylococcus aureus. A sorologia para o HIV resultou negativa, mostrando-se positiva para 0 HTLV-1 (EUSA e Western blot) na criança e em seus pais. Na primeira internação, a criança recebeu antibioticoterapia, hidrocortisona creme e umectação com creme hidratante, com boa resposta clínica. Entretanto, houve recidiva, o que justificou nova internação. Após melhora significativa, ela recebeu alta hospitalar e não retornou ao serviço por ter se mudado com os pais para outro estado.

Além de um provável papel prodrômico na ATL, suspeitase de que a DI possa predizer também o desenvolvimento de HAM/TSP ${ }^{51}$. Discute-se se um mesmo fator predisponente (e.g., HLA DRB1*DQB1*) poderia, simultaneamente, determinar 0



Figura 1 - Dermatite infecciosa em uma criança de dois anos de idade, infectada pelo HTLV-1. Lesões eczematosas observadas predominantemente no couro cabeludo, face e nas regiões inguinal, axilar e cervical.

desenvolvimento dessa dermatose e da HAM/TSP57. Estudos epidemiológicos e moleculares são necessários para esclarecer se a DI constitui unicamente uma forma grave de dermatite generalizada associada ao HTLV-1 ou se ela representa, também, um marcador do desenvolvimento de patologias mais graves associadas ao vírus $^{99}$.

4- Outras lesões cutâneas descritas em pacientes infectados pelo HTLV1: a presença de lesões cutâneas nos pacientes infectados pelo HTLV-1 independe da coexistência de ATLL ou de HAM/TSP. Nesse sentido, Gonçalves e colaboradores, estudando candidatos à doação de sangue pela Fundação Hemominas de Minas Gerais, compararam o exame dermatológico de indivíduos infectados pelo HTLV-1 com o de doadores sem essa infecção. Os autores concluíram que os portadores do vírus apresentam lesões dermatológicas com frequêencia significativamente elevada, com destaque para ictiose adquirida idiopática, dermatite seborréica e, infecção pela Tinea pedis ( Figuras 2 e 3) . Houve associação fraca da infecção pelo HTLVI com vitiligo e onicomicose. Outro achado relevante desse estudo foi 0 de que a presença do HTLV-1 na pele, pesquisada pela técnica de PCR, mostrou-se mais comum conforme a maior intensidade da lesão cutânea observada, clínica e/ou histológica ${ }^{31}$.

Estudando 30 membros de três gerações de uma família, 15 (50\%) deles infectados pelo HTLV-1, Nobre demonstrou que xerodermia e ictiose adquirida ocorreram exclusivamente entre os infectados pelo vírus. Adicionalmente, dois pacientes infectados apresentavam cicatriz de herpes zoster, condição geralmente associada à imunodeficiência. Na pesquisa do HTIV1 na pele através da técnica de PCR, encontrou-se material genético do vírus nos 13 pacientes com sorologia positiva em que 0 exame foi realizado (dados não publicados). Quanto ao herpes zoster, outros relatos na literatura sugerem a ocorrência dessa virose em pacientes infectados pelo HTLV-1, mesmo quando eles não são portadores de ATL ${ }^{87}$.

Dentre as infecções bacterianas relatadas, destaca-se a DI, geralmente associada à infecção pelo Staphylococcus aureus e/ou Streptococcus beta hemolítico. Outro exemplo de infecção bacteriana descrito é a foliculite decalvante, embora haja apenas relatos isolados ${ }^{3}$. 


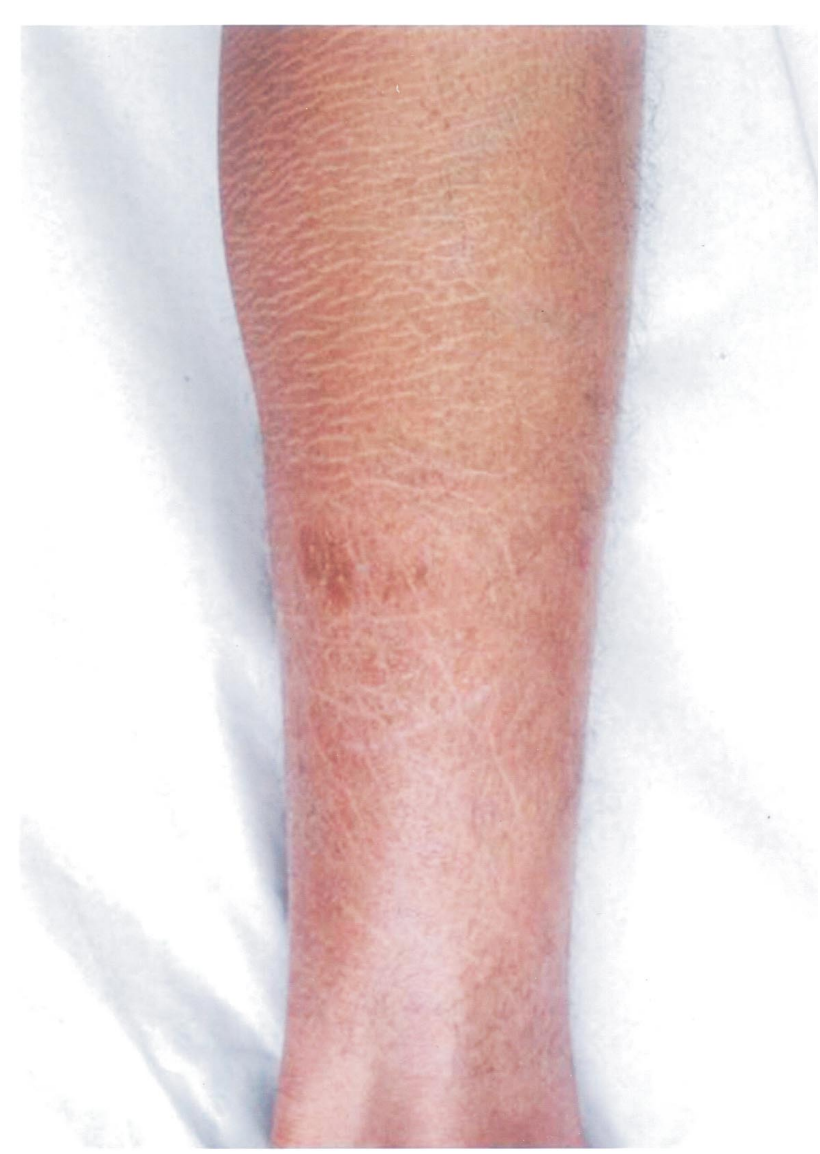

Figura 2 - Ictiose adquirida idiopática, observada na perna de um paciente portador assintomático do HTLV-1.

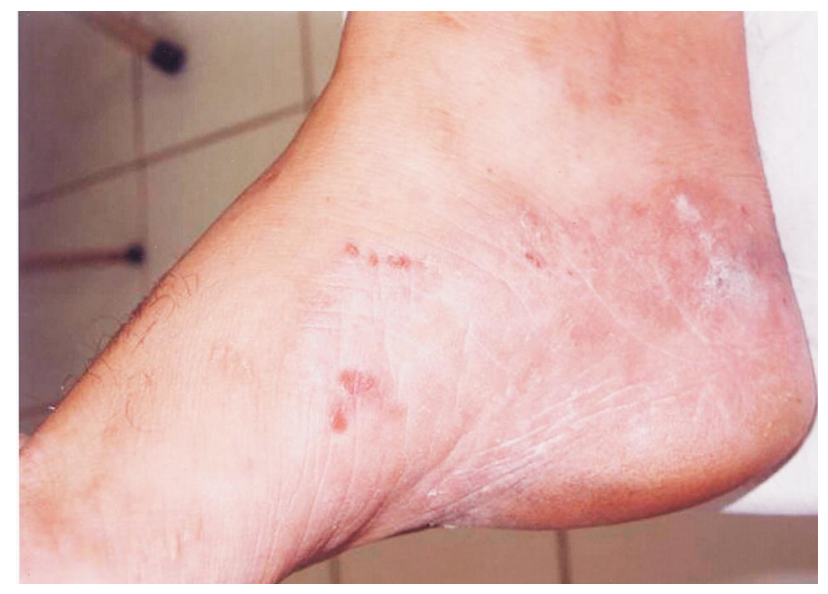

Figura 3 - Tinea pedis, diagnosticada em um outro portador assintomático do HTLV-1.

Uma outra evidência de que a infecção pelo HTLV-1 associase ao desenvolvimento de lesões cutâneas refere-se ao fato de que pacientes atendidos em clínicas dermatológicas apresentam maior prevalência da infecção por esse vírus. Na Nigéria, Olumide e colaboradores ${ }^{72}$ demonstraram prevalências de soropositividade de 35\% a 50\% em uma clínica dermatológica e de doenças sexualmente transmissíveis, utilizando ELISA e Western blot. Na Índia, encontrou-se prevalência de 4,4\% de 45 pacientes atendidos em clínica de dermatologia ${ }^{1}$. Nobre encontrou prevalência de 0,73\% para o HTLV-1 em 1229 pacientes atendidos em clínica dermatológica da cidade de Belo Horizonte, em Minas Gerais.
A prevalência da infecção em candidatos à doação de sangue atendidos no mesmo período pela Fundação Hemominas foi de $0,01 \%$ (dados não publicados).

As características de algumas outras dermatopatias descritas em pacientes infectados pelo HTLV-1, inclusive naqueles sem ATLL ou HAM/TSP, são descritas a seguir:

\section{ESCABIOSE}

Acredita-se que, a escabiose, ectoparasitose causada pelo ácaro Sarcoptes scabiei, ocorra nos pacientes infectados pelo HTLV-1 predominantemente devido à imunodepressão determinada pelo vírus ${ }^{87}$. A escabiose em geral cursa com manifestações discretas, mas pode se agravar em pacientes infectados pelo HTLV-1. A forma grave dessa doença é chamada de escabiose crostosa ou norueguesa ${ }^{18}$. Tem-se demonstrado, inclusive, que a infecção pelo HTLV-1 determina 0 desenvolvimento dessas apresentações independentemente da presença de ATLL 1212270 .

№ Japão, Suzumiya e colaboradores descreveram um caso de escabiose norueguesa em paciente com 0 diagnóstico de ATLL $^{95}$. Del Giudice e colaboradores ${ }^{23}$ relataram seis casos de escabiose norueguesa na Guiana Francesa, ocorrendo em pacientes portadores do HTLV-1, quatro deles com diagnóstico de ATL. Os dois pacientes sem ATLL apresentavam, respectivamente, estrongiloidíase recorrente e tuberculose pulmonar.

Quanto à ocorrência de casos em portadores do vírus sem ATL, considerados assintomáticos, Cordoliani e colaboradores ${ }^{21}$ relataram 0 caso de uma paciente africana que apresentou escabiose norueguesa e Tinea corporis difusa. Daisley e colaboradores ${ }^{22}$ reportaram outro caso, com escabiose recidivante associada a Tinea corporis e Tinea unguium, em mulher jovem, HTLV-1 positiva. No mesmo ano, Mollison e colaboradores $^{70}$ encontraram soropositividade para 0 HTLV-1 em cinco aborígines australianos portadores de escabiose norueguesa. Por outro lado, apenas seis de 19 pacientes com outras condições clínicas revelaram-se infectados. Mais recentemente, no Brasil, Brites e colaboradore ${ }^{12}{ }^{2}$ revisaram 91 casos de escabiose, ocorridos no estado da Bahia, e encontraram prevalências elevadas da infecção pelo HTLV-1 (32\%) , HIV (50\%) ou pelos dois vírus (20\%) nestes pacientes. Demonstraram ainda que os casos mais graves de escabiose estavam significativamente associados à infecção pelo HTLV-1.

\section{ICTIOSE ADQUIRIDA}

Aictiose representa a forma mais intensa e de difícil controle da xerose cutânea. Asua etiologia e a patogênese não se encontram definidas. Há duas formas principais de ictiose, a congênita e a adquirida. A ictiose adquirida predomina na idade adulta e pode se associar a várias doenças, como linfoma, hipotireoidismo, hanseníase e lupo eritematoso sistêmico. Vários medicamentos também têm sido implicados na etiologia dessa dermatose. Em outro grupo de casos ela é considerada idiopática². 
Xerose cutânea e ictiose constituem achados comumente descritos em pacientes infectados pelo HTLV-1, notadamente nos portadores de HAM/TSP36 ${ }^{60}$. As lesões predominam na face lateral das pernas, nos flancos e nos braços, mas podem acometer todo o corpo (Figura 2). Nos pacientes com HAM/TSP, a hiperceratose apresentada parece ocorrer por retenção (em contraposição ao tipo hiper-proliferativo, observado em outras doenças) ${ }^{67}$.

Kaplan e colaboradores demonstraram maior freqüência de ictiose adquirida em pacientes infectados pelo HIV quando estes são co-infectados pelo HTLV-2 ${ }^{43}$. A ictiose adquirida foi apontada por Gonçalves e colaboradores como sendo condição associada à sorologia positiva para o HTLV1 em candidatos à doação de sangue ${ }^{31}$. Mais recentemente, estudando 30 membros de uma família no Brasil, Nobre e colaboradores demonstraram predomínio de xerodermia e de ictiose adquirida dentre as dermatoses encontradas nos indivíduos infectados pelo HTLV-1, quando comparados aos seus familiares soronegativos ( dados não publicados).

\section{DERMATITE SEBORREICA}

Adermatite seborréica tem sido comumente demonstrada em pacientes infectados pelo HTLV-1, na forma de relatos isolados ${ }^{14}$ ou em estudos controlados de candidatos a doadores de sangue ${ }^{30}{ }^{31}$. Especula-se que, à semelhança do que ocorre na infecção pelo HIV, a imunodepressão celular possa predispor a uma maior frequiência de dermatite seborréica observada nos pacientes infectados pelo HTLV-1. Além disso, a dermatite seborréica guarda diversas semelhanças com a DI ${ }^{52}$. Em uma coorte de 308 crianças, 28 infectadas pelo HTLV-1 e 280 sem a infecção, Maloney e colaboradores ${ }^{64}$ demonstraram que as crianças infectadas apresentam frequência cinco vezes maior de dermatite seborréica e duas vezes maior de eczema, quando comparadas com crianças não infectadas. Assim, todo paciente com dermatite seborréica mais extensa, sobretudo se for criança, deveria submeter-se à sorologia para a infecção pelo HTLV-1.

\section{VITILIG0}

Há várias citações de vitiligo ocorrendo em pacientes infectados pelo HTLV-1, com e sem HAM/TSP ou ATLL ${ }^{14} 3188$. Dentre nove pacientes com sorologia positiva para 0 HTLV-1, identificados em um grupo de 1229 pacientes atendidos em clínica dermatológica, Nobre encontrou dois casos de vitiligo ( dados não publicados). Entretanto, Grimes e colaboradores ${ }^{32}$ encontraram apenas o citomegalovírus (38\% dos casos), ao pesquisarem diversos agentes virais, pela técnica de PCR, na pele de 29 pacientes com vitiligo. A pesquisa do HTLV-1 revelouse negativa, na pele, em todos os casos.

\section{MICOSES CUTÂNEAS}

À semelhança da escabiose, as micoses cutâneas, notadamente as dermatofitoses, ocorrem com maior freqüência nos pacientes infectados pelo HTLV-1 (Figura 3). 0 mecanismo sugerido é o da imunossupressão celular determinada pelo vírus ${ }^{33}{ }^{87}$. Casos de dermatofitose extensa associada à escabiose norueguesa foram descritos ${ }^{212}$. Além disso, portadores assintomáticos do HTLV-1 apresentam maior freqüência de dermatofitoses ${ }^{31}$.

\section{CONCLUSÃO}

Diversas lesões cutâneas são observadas nos indivíduos infectados pelo HTLV-1. A despeito de predominarem nos pacientes com ATLLou HAM/TSP, observa-se, de modo crescente que essas lesões também ocorrem com freqüência aumentada nos portadores do vírus. A identificação e 0 estudo das dermatopatias mais prevalentes em pacientes infectados pelo HTLV-1, assim como a eventual definição de outras lesões específicas (como a dermatite infecciosa), auxiliará no entendimento da fisiopatologia, assim como na identificação precoce dessa infecção.

\section{REFERÊNCIAS BIBLIOGRÁFICAS}

1. Ajithkumar K, Romalingan S, Kannangai R, Prakash KJ. Human T lymphotropic vírus-1 (HTLV-1) infection in patients with unclassifiable dermatitis in central Kerala, South India: a preliminary study. Sexually Transmitted infections 78: 434, 2002.

2. Aram H. Acquired ichthyosis and related conditions. International Journal of Dermatology 23: 458-461, 1984.

3. Araújo AQC, Andrade-Serpa MJ, Paulo-filho TA, Rodrigues MT, Prado LAF. Folliculitis decalvans and human T cell lymphotropic vírus type I associated myelopathy/tropical spastic paraparesis. Clinical Infectious Diseases 20: 696-699, 1995.

4. Bazarbachi A, Soriano V, Pawson R, Vallejo A, Moudjil T, Matutes E, Peries J, Molina A, The H, Shuz TF, Catovsky D, Gill PS. Mycosis fungoides and Sézary syndrome are not associated with HTLV-1 infection: an international study. British Journal of Haematology 98: 927-933, 1997.

5. Benhur Junior A, Serufo JC, Lambertucci JR. Pulmonary strongyloidiasis. Revista da Sociedade Brasileira de Medicina Tropical 37: 359-360, 2004.

6. Biggar RJ, Saxinger C, Gardiner C, Collins WE, Levine PH, Clark JW, Nkrumah FK, Blattner WA. Type 1 HTLV antibody in urban and rural Ghana, West Africa. International Journal of Cancer 34: 215-219, 1984.

7. Bittencourt AL, Barbosa HS, Brites C, Ferraz N, Freitas V, Pereira Filho, CS, Harrington Jr W. Clinicopathological aspects of HTLV-I positive and negative cutaneous T-cell lymphoma. European Journal of Dermatology 7: 283-289, 1997.

8. Bittencourt AL, Fernandes DJ, Sampaio Filho C, Moreira Jr ED, Ribeiro TT, Harrington Jr W. HTLV-I associated cutaneous T-cell lymphoma: report of a case with atypical clinical presentation. Memórias do Instituto Oswaldo Cruz 89: 59-61, 1994.

9. Blank A., Herrera M, Lourido MA, Rueda R, Blank M. Infective dermatitis in Colombia. Lancet 346: 710, 1995.

10. Boni R, Davis-Daneshfar A., Burg G, Fuchs D, Wood GS. No detection of HTLV-I proviral DNA in lesional skin biopsies from Swiss and German patients with cutaneous T-cell lymphoma. British Journal of Dermatology 134: 282-284, 1996.

11. Brites C, Maciel E, Espinheira L. Strongyloidiasis as an opportunistic infection in a HAM/TSP patient. Brazilian Journal of Infectious Diseases 3: 23-27, 1999.

12. Brites C, Weyl M, Pedroso C, Badaró R. Severe and Norwegian scabies are strongly associated with retroviral (HIV/HTLV-I) infection in Bahia, Brazil. AIDS 16: 1292-1293, 2002. 
13. Bunker CB, Whittaker S, Luzzato L, Gore ME, Rustin MHA, Smith NP, Levine GM. Indolent cutaneous prodrome of fatal HTLV-I infection. Lancet 335: 426, 1990.

14. Carnaúba Jr D, Braga Jr, JWR, Oliveira LDP, Canuto CPB, Diegues JF, Moraes AL. Manifestações dermatológicas em pacientes com mielopatia associada ao HTLV-I. In: Simpósio Internacional Sobre HTLV-I/II no Brasil, 4, Belo Horizonte. Resumos dos trabalhos apresentados. Belo Horizonte: [s.n.], p.49, 1996.

15. Carnaúba Jr D, Bittencourt A, Brites C. Atypical presentation of syphilis in an HTLV-I infected patient. The Brazilian Journal of Infectious Diseases 7: 271-277, 2003

16. Carneiro-Proietti ABF, Ribas JG, Catalan-Soares B, Martins M, Brito-Melo GEA, Martins-Filho 0, Araujo AQC, Galvão-Castro B, Pombo de Oliveira MS, Guedes AC, Proietti FA. Infecção e doença pelos vírus linfotrópicos humanos de células T ( HTLV-I/II) no Brasil. Revista da Sociedade Brasileira de Medicina Tropical 35: 499-508, 2002.

17. Carneiro-Proietti ABF, Catalan-Soares B, Proietti FA. Human T-cell lymphotropic vírus in South America: should it be a public health concern? Journal of Biological Sciences 9: 587-595, 2002.

18. Chosidow 0. Scabies and pediculosis. Lancet 355: 819-826, 2000.

19. Coffin JM. Retroviridae: the víruses and their replication. In: Fields BN, Knipe DM, Howley PM ( eds) Fields virology. $3^{\text {rd }}$ ed. Philadelphia: LippincottRaven Publishers, v.2, cap.58, p.1767-1847, 1996.

20. Constantine NT. Serologic tests for the retrovíruses: approaching a decade of evolution. AIDS 7: 1-13, 1993.

21. Cordoliani F, Vasseur E, Baccard M, Fournier S, Feuilhade-Cheuvin M, Tancrede E, Morel P. Ivermectin-responsive crusted scabies in HTLV1 carrier. Dermatology 192:351-352, 1996.

22. Daisley H, Charles W, Suite M. Crusted (Norwegian) scabies as a prediagnostic indicator for HTLV-1 infection. Transactions of Royal Society of Tropical Medicine and Hygiene 87: 295, 1993.

23. Del Giudice P, Sainte Marie D, Gérard Y, Couppié P, Pradinaud R. Is crusted ( Norwegian) scabies a marker of adult $\mathrm{T}$ cell leucemia/lymphoma in human T lymphotropic vírus type 1-seropositive patients? Journal of Infectious Diseases 176: 1090-1092, 1997.

24. Dosaka N, Tanaka T, Miyachi Y, Ymamura S, Kakizuka A. Examination of HTLV-I integration in the skin lesions of various types of adult T-cell leukemia (ATL) : independence of cutaneous-type ATL confirmed by southern blot analysis. Journal of Investigative Dermatology 96: 196-200, 1991

25. Dourado I, Alcantara LC, Barreto ML, Gloria Teixeira M, Galvao-Castro B. HTLV-I in the general population of Salvador, Brazil: a city with African ethnic and sociodemographic characteristics. Journal of Acquired Immune Deficiency Syndrome 34: 527-531, 2003.

26. Ehrlich GD, Glaser JB, Lavigne K, Quan D, Mildvan D, Sninsky JJ, Kwok S, Papsidero L, Poiesz BJ. Prevalence of human T-cell leukemia/lymphoma vírus (HTLV) type II infection among high-risk individuals: type-specific identification of HTLVs by polymerase chain reaction. Blood 74: 1658-1664, 1989.

27. Gessain A, Barin F, Vernant JC, Gout O, Maurs L, Calender A., Thé G. Antibodies to human T-lymphotropic vírus type-I in patients with tropical spastic paraparesis. Lancet 24: 407-409, 1985.

28. Gessain A, Caumes E, Feyeux C, Dàgay MF, Capesius C, Gentilini M, Morel P. The cutaneous form of adult T-cell leukemia/lymphoma in a woman from Ivore Coast. Cancer 69: 1362-1367, 1992

29. Gessain A, Moulonguet I, Flageul B, Perrin P, Capesius C, D'agay MF, Gisselbrecht C, Sigaux F, Civatte J. Cutaneous type of adult T cell leukemia/ lymphoma in a Franch West Indian woman. Journal of the American Academy of Dermatology 23: 994-1.000, 1990.

30. Gonçalves DU, Guedes AC, Carneiro-Proietti A.B, Lambertucci J.R. HTLV-I associated infective dermatitis may be an indolent HTLV-I associated lymphoma. Brazillian Journal of Infectious Diseases:100-102, 2000.

31. Gonçalves DU, Guedes AC, Proietti AB, Martins ML, Proietti FA, Lambertucci JR. Interdisciplinary HTLV-1/2 Research Group. Dermatologic lesions in asymptomatic blood donors seropositive for human T cell lymphotropic vírus type-1. American Journal of Tropical Medicine and Hygiene 68: 562-5, 2003.
32. Grimes PE, Sevall JS, Vojdani A. Cytomegalovírus DNA identified in skin biopsy specimens of patients with vitiligo. Journal of the American Academy of Dermatology 35: 21-26, 1996.

33. Grossman ME, Pappert AS, Garzon MV, Silvers DN. Invasive Trichophyton rubrum infection in the immunocompromised host: report of three cases. Journal of the American Academy of Dermatology 33: 315-318, 1995.

34. Hall WW, Ishak R, Zhu SW, Novoa P, Eiraku N, Takahashi H, Ferreira MDAC Azevedo V, Ishak M0, Ferreira ODAC, Monken C, Kurata T. Human T lymphotropic vírus type II ( HTLV-II) : epidemiology, molecular properties, and clinical features of infection. Journal of Acquired Immune Deficiency Syndrome and Human Retrovirology 13 ( suppl 1) S204-214, 1996.

35. Hanchard B, LaGrenade L, Carberry C, Fletcher V, Williams E, Cranston B, Blattner W, Manns A. Childhood infective dermatitis evolving into adult Tcell leukaemia after 17 years. Lancet 338: 1593-1594, 1991

36. Hashiguchi T, Osame M, Arimura K, Fijiyama J, Furukawa Y, Kubota R, Koreeda Y, Maruyama I, Matsumoto M, Tashiro M, Sato E. Skin manifestations in HTLV-I associated myelopathy (HAM): xerosis and erythema. In: Róman CG, Vernanti C, Osame M (eds) HTLV-I and the nervous system. Alan R. Liss, New York, p.443-448, 1989.

37. Higaki Y, Mizushima J, Kawashima M, Motoji T, Mizoguchi H. Cutaneous granulomas associated with adult T-cell leukemia/lymphoma. Dermatology 206: 157-160, 2003.

38. Hinuma Y, Komoda H, Chosa T, Kondo T, Kohakura M, Takenaka T, Kikuchi M, Ichimaru M, Yunoki, K, Sato I, Matsuo R, Takiuchi Y, Uchino H, Hanaoka M. Antibodies to adult T-cell leukemia-vírus associated antigen (ATLA) in sera from patients with ATL and controls in Japan: a nation-wide seroepidemiologic study. International Journal of Cancer 29: 631-635, 1982.

39. Hinuma Y, Nagata K, Hanaoka M, Nakai M, Matsumoto T, Kinoshita KI, Shibarawa S, Miyoshi I. Adult T-cell leukemia: antigen in an ATL cell line and detection of antibodies to the antigen in human sera. Proceedings of the National Academy of Science 78: 6476-6480, 1981.

40. Ishida T, Yamamoto K, Omoto K, Iwanaga M, Osato T, Hinuma Y. Prevalence of a human retrovírus in native Japanese: evidence for a possible ancient origin. Journal of Infection 11:153-157, 1985.

41. Kajiyama W, Kashiwagi S, Ikematsu H, Hayashi J, Nomura H, Okochi K. Intrafamilial transmission of adult $\mathrm{T}$ cell leukemia vírus. Journal of Infectious Diseases 154: 851-857, 1986.

42. Kalyanaraman VS, Sarndadharan MG, Robert-Guroff M, Miyoshi I, Blayney D, Golde D, Gallo RC. A new subtype of human T-cell leukemia vírus ( HTLVII) associated with a T-cell variant of hairy cell leukemia. Science 218 : 571-573, 1982

43. Kaplan MH, Sadick NS, McNutt S, Talmor M, Coronesi M, HALL W. Acquired ichthyosis in concomitant HIV-1 and HTLV-II infection: a new association with intravenous drug abuse. Journal of the American Academy of Dermatology 29: 701-708, 1993.

44. Katsuki T, Katsuki K, Imai J, Hinuma Y. Immune suppression in healthy carriers of adult T-cell leukemia retrovírus ( HTLV-I) : impairment of T-cell control of Epstein-Barr vírus-infected B-cells. Japanese Journal of Cancer Research 78: 639-642: 1987.

45. Kerl H, Cerroni L, Burg G. The morphological spectrum of T-cell lymphomas of the skin: a proposal for a new classification. Seminars in Diagnostic Pathology 8: 55-61, 1991.

46. Khan JM, Sebenik M, Zucker-Franklin, D. Localization of human T-cell lymphotropic vírus-1 tax proviral sequences in skin biopsies of patients with mycosis fungoides by in situ polymerase chain reaction. Journal of Investigative Dermatology 106: 667-672, 1996.

47. Kikuchi A, Nishikawa T, Ikeda Y, Yamaguchi K. Absence of human Tlymphotropic virus type I in Japanese patients with cutaneous T-cell lymphoma. Blood 89: 1529-1532, 1997.

48. Kirsner RS, Federman DG. Cutaneous clues to systemic disease. Postgraduate Medicine 101: 137-139, 1997.

49. Kuwabara H, Nagai M, Hojo W, Yamada A, Miki H, Takeuchi H, Teranishi K, Matsuda K, Uchida Y, Nakashima K, Sasaki M, Sepp R. Human T lymphotropic vírus type I associated myelopathy with pulmonary and cutaneous lesions. Journal of Clinical Pathology 46: 273-275, 1993. 
50. La Grenade L. Infective dermatitis of Jamaican children 1966-1991. West Indian Medical Journal 41 (suppl) : 33, 1992.

51. La Grenade L. Tropical spastic paraparesis occurring in HTLV-1-associated infective dermatitis: report on two cases. West Indian Medical Journal 44: 34-35, 1995.

52. La Grenade L. HTLV-I associated infective dermatitis: past, present and future. Journal of Acquired Immune Deficiency Syndromes Human Retrovírus 13: 46-49, 1996.

53. La Grenade L. Manifestações dermatológicas do HTLV-I. In: Proietti, ABFC (ed) HTLV-I/II. Cadernos Hemominas, vol. XI. Cap. 4, p139-146, 2000.

54. La Grenade L, Hanchard B, Fletcher V, Cranston B, Blattner W. Infective dermatitis of Jamaican children: a marker for HTLV-I infection. Lancet 336: 1.345-1.347, 1990.

55. La Grenade L, Manns A, Fletcher V, Carberry C, Hanchard B, Maloney E, Cranston B, Williams NP, Wilks R, Kang EC, Blattner WA. Clinical, Pathologic, and immunologic features of human T-lymphotropic vírus type I-associated infective dermatitis in children. Archives of Dermatology 134: 439-444, 1998.

56. La Grenade L, Scwartz RA, Janniger C.K. Childhood dermatitis in the tropics: with special emphasis on infective dermatitis, a marker for infection with human T-cell leukemia vírus-I. Cutis 58: 115-118, 1996.

57. La Grenade L, Sonoda S, Miller W, Rodgers-Johnson P, Hanchard B, Cranston B, Fujiyoshi T, Yashiki S, Blank M, Gibbs CJ, Manns A. HLA DRB1*DQB1* haplotype in HTLV-I-associated familial infective dermatitis may predict development of HTLV-I-associated myelopathy/tropical spastic paraparesis. American Journal of Medical Genetics 61: 37-41, 1996.

58. Lambertucci JR, Leao FC, Barbosa AJ. Gastric strongyloidiasis and infection by the human $T$ cell lymphotropic vírus type 1 (HTLV-1). Revista da Sociedade Brasileira de Medicina Tropical 36: 541-542, 2003.

59. Lenzi MER, Araujo AQC, Maya TC, Serapião MJ, Leite ACC, Schor D, AndradeSerpa MJ. Dermatite infectiva associada ao HTLV-I: relato de caso. Anais Brasileiros de Dermatologia 71: 115-118, 1996.

60. Lenzi MER, Cuzzi-Maya T, Oliveira ALA, Andrade-Serpa MJ, Araujo AQC. Dermatological findings of human T lymphotropic vírus type I (HTLV-I) associated myelopathy/tropical spastic paraparesis. Clinical Infectious Diseases 36: 507-513, 2003.

61. Levine PH, Cleghorn F, Manns A, Jaffe ES, Navarro-Roman L, Blattner WA, Hanchard B. Oliveira MS, Matutes E, Catovsky D, Shimoyama M, Tajima K, Sonoda S, Yamaguchi K, Takatsuki K. Adult T-cell leukemia/lymphoma: a working point-score classification for epidemiological studies. International Journal of Cancer 59: 491-493, 1994.

62. Lisby G, Reitz MS, Vejlsgaard GL. No detection of HTLV-I DNA in punch skin biopsies from patients with cutaneous T-cell lymphoma by the polymerase chain reaction. Journal of Investigative Dermatology 98: 417-20, 1992.

63. Madeleine MM, Wiktor SZ, Goedert JJ. HTLV-I and HTLV-II world-wilde distribution: reanalysis of 4,832 immunoblot results. International Journal of Cancer 54: 258-260, 1993.

64. Maloney EM, Wiktor SZ, Palmer P, Cranton B, Pate EJ, Cohn S, Kim N, Miley W, Thomas TL, Blattner WA, Hanchard B. A cohort study of health effects of human T-lymphotropic vírus type I infection in Jamaican children. Pediatrics 112: 136-142, 2003.

65. Manns A, Hisada M, La Grenade L. Human T-lymphotopic vírus type I infection. Lancet 353: 351-358, 1999.

66. Marsh B.J. Infectious complications of human leukemia/lymphoma vírus type I infection. Clinical Infectious Diseases 23: 138-145, 1996.

67. Milagres SP, Sanches JA, Milagres ACP, Valente NYS. Histopathology and immunohistochemical assessment of acquired ichthyosis in patients with human T-cell lymphotropic vírus type I-associated myelopathy. British Journal of Dermatology 149: 776-781, 2003.

68. Mochizuki M, Watanabe T, Yamaguchi K, Yoshimura K, Nakashima S, Shirao M, Araki S, Takatsuki K., Mori S, Miyata N. Uveitis associated with human T-cell lymphotropic vírus type 1. American Journal of Ophtalmology 114: 123-129, 1992.

69. Mochizuchi M, Yamaguchi K, Takatsuki K, Watanabe T, Mori S, Tajim K. HTLV-I and uveitis [letter]. Lancet 339: 1110, 1992.
70. Mollison LC, Lo STH, Marning G. HTLV-I and scabies in Australian aborigines. Lancet 341: 1.281-1.283, 1993.

71. Nakada K, Yamaguchi K, Furugen S, Nakasone T, Nakasone K, Oshiro Y, Kohakura M, Hinuma Y, Seiki M, Yoshida M, Matutes E, Catovsky D, Ishi T, Takatsuki M. Monoclonal integration of HTLV-I proviral DNA in patients with strongyloidiasis. International Journal of Cancer 40: 145-148, 1987.

72. Olumide YM, Dada AJ, Sogbanmu IB, Aruna GA. Seroprevalence study of HIV-I, HIV-II and HTLV-I among patients at the Dermato-Venereology Clinic of the Lagos University Teaching Hospital. International Journal of Dermatology 36:741-744, 1997.

73. Osame M, Usuku K, Izumo S, Ijishi N, Amitani H, Igata A, Matsumoto M. HTLV-I associated myelopathy, a new clinical entity. Lancet 1: 1031-1032, 1986.

74. Pancake BA, Zucker-Franklin D. The difficulty of detecting HTLV-I proviral sequences in patients with mycosis fungoides. Journal of Acquired Immune Deficiency Syndromes and Human Retrovirology 13: 314-319, 1996.

75. Pancake BA, Zucker-Franklin D, Coutavas EE. The cutaneous T cell lymphoma, mycosis fungoides, is a human $\mathrm{T}$ cell lymphotropic vírusassociated disease. A study of 50 patients. Journal of Clinical Investigation 95: 547-554, 1995.

76. Pancake E, Zucker-Franklin D. HTLV tax and mysosis fungoides. New England Journal of Medicine 329: 580, 1993.

77. Patey 0, Gessain A, Breuil J, Courillon-Mallet A, Aniel MT, Miclea JM, Roucayrol AM, Sigaux F, Lafaix C. Seven years of recurrent severe strongyloidiasis in an HTLV-I-infected man who developed adult T-cell leukemia. AIDS 6: 575-579, 1992.

78. Pfeilsticker OMC. Pesquisa do vírus HTLV-I em biópsias de pele de indivíduos soropositivos: aspectos ultra-estruturais e moleculares. Dissertação (Mestrado) - Programa de Pós-Graduação em Microbiologia da Universidade Federal de Minas Gerais, Belo Horizonte, 2002.

79. Poiesz BJ, Ruscetti FW, Gazdar AF, Bunn PA, Minna JD, Gallo RC. Detection and isolation of type-C retrovirus particles from fresh and cultured lymphocytes of a patient with cutaneous T cell lymphoma. Proceedings of the National Academy of Sciences 77: 7415-7419, 1980.

80. Poiesz BJ, Ruscetti F, Reitz MS, Kalyanaraman VS, Gallo R. Isolation of a new type $C$ retrovírus ( HTLV) in primary uncultured cells of a patient with Sézary T cell leukemia. Nature 294: 268-271, 1981.

81. Popovic M, Flomenberg N, Volkman DJ, Mann D, Fauci AS, Dupont B, Gallo R. Alteration of T-cell functions by infection with HTLV-I or HTLV-II. Science 226: 459-461, 1984.

82. Porto MAF, Muniz A, Oliveira Jr, J, Carvalho EM. Implicações clínicas e imunológicas da associação entre o HTLV-I e a estrongiloidíase. Revista da Sociedade Brasileira de Medicina Tropical 35: 641-649, 2002.

83. Proietti FA, Lima-Martins MVC, Passos VMA, Brener S, Carneiro-Proietti AB. HTLV-I/II seropositivity among elegible blood donors from Minas Gerais State, Brasil. Vox Sanguinis 67: 77, 1994.

84. Reeves WC, Saxinger C, Brener MM, Quiroz E, Clark JW, Hoh MH, Blattner WA. Human T cell lymphotropic vírus type-I (HTLV-I) seroepidemiology and risk factors in metropolitan Panama. American Journal of Epidemiology 127: 532-539, 1988

85. Ribas JGR, Melo GCN. Mielopatia associada ao vírus linfotrópico humano de células T do tipo 1 (HTLV-1). Revista da Sociedade Brasileira de Medicina Tropical 35: 377-384, 2002.

86. Róman G, Osame M. Identity of HTLV-I-associated tropical spastic paraparesis and HTLV-I-associated myelopathy. Lancet 1: 651, 1998.

87. Rueda R, Blank A. HTLV-I associated cutaneous manifestations. In: Zaninovic $\mathrm{V}$ (ed) HTLV - thruths and questions. Cali: Feriva, p. 212-222, 1996.

88. Salazar-Grueso EF, Holzer TJ, Gutierrez RA, Casey JM, Desai SM, Devare SG, Dawson G, Roos RP. Familial spastic paraparesis syndrome associated with HTLV-I infection. New England Journal of Medicine 323: 732-737, 1990.

89. Sarim SG, Fang C, Willians A. Retroviral infections transmited by blood transfusion. Yale Journal of Biology and Medicine 63: 353-360, 1990.

90. Setoyama M, Kerdel FA, Elgard G, Kanzaki T, Byrnes J.J. Detection of HTLV1 by polymerase chain reaction in situ hybridization in adult T-cell leukemia/lymphoma. American Journal of Pathology 152: 683-689, 1998. 
91. Setoyama M, Mizoguchi S, Kanzaki T. Prurigo as a clinical prodrome to adult T-cell leukemia/lymphoma. British Journal of Dermatology 138: 137 $140,1998$.

92. Sharata HH and Colvin JH, Fuhiwara K, Goldman B, Hashimoto K. Cutaneous and neurologic disease associated with HTLV-I infection. Journal of the American Academy of Dermatology 36 ( 5 Pt 2) : 869-871, 1997.

93. Shimoyama M and Members of the Lymphoma Study Group (1984-87). Diagnostic criteria and classification of clinical subtypes of adult T-cell leukaemia/lymphoma. British Journal of Haemathology 79: 428-437, 1991.

94. Siegel RS, Pamdolfino T, Guitard J, Rosen S, Kuzel TM. Primary cutaneous T-cell lymphoma: review and current concepts. Journal of Clinical Oncology 18: 2.908-2.925, 2000

95. Suzumiya J, Sumiyoshi A, Kuroki Y, Onoue S. Crusted (Norwegian) scabies with adult T-cell leukemia. Archives of Dermatology 121: 903-904, 1985.

96. Sweet RD. A pattern of eczema in Jamaica. British Journal of Dermatology 78, 93-100, 1966.

97. Tachibana N, Okayama A, Ishizaki J, Yokota T, Shishime E, Murai K, Shiori S, Tsuda K, Essesx M, Mueller N. Suppression of tuberculin skin reaction in healthy HTLV-I carriers from Japan. International Journal of Cancer 42, 829-831, 1988.

98. ThariatJ. Cutaneous manifestations of the leukemia-lymphoma of HTLV 1: a case report. Medicine Tropicale 61: 173-176, 2001.
99. Tschachler E, Franchini G. Infective Dermatitis. A pabulum for human Tlymphotropic vírus type I leukemogenesis? Archives of Dermatology 134: 487-488, 1998.

100. Tsukasaki K, Yamada Y, Ikeda S, Tomonaga M. Infective dermatitis among patients with ATL in Japan. International Journal of Cancer 57: 293, 1994.

102. Uchiyama T, Yodoi J, Sagawa K, Takatsuki K, Uchino H. Adult T-cell leukemia: clinical and hematologic features of 16 cases. Blood 50: 481492, 1977.

103. Yamaguchi K. Human T-Lymphotropic Vírus Type I in Japan. Lancet 43: 213-216, 1994.

104. Yoshida M, Seiki M, Yamaguchi K, Takatsuki K. Monoclonal integration of human T-cell leukaemia provírus in all primary tumors of adult T-cell leukaemia suggests causative role of human T-cell leukaemia vírus in the disease. Proceedings of the National Academy of Sciences 81: 2534-2537, 1984.

105. Walshe MM. Infective dermatitis in Jamaican children. British Journal of Dermatology 79: 229-236, 1967.

106. Whittaker SJ, Ng YL, Levene G, Mcgibbon DH, Smith NP. HTLV-1-associated cutaneous disease: a clinicopathological and molecular study of patients from the U.K. British Journal of Dermatology 128: 483-492, 1992.

107. Wood GS, Schaffer JM, Boni R, Dummer R, Burg G, Takeshita M, Kikuchi M. No evidence of HTLV-1 proviral integration in lymphoproliferative disorders associated with cutaneous T-cell lymphoma. American Journal of Pathology 150:667-673, 1997. 